\title{
Informatisation de la méthode Bachet
}

\author{
J. Miquel A. Lebossé \\ Laboratoire national d'hydraulique, EDF Chatou
}

\section{Principes}

\subsection{Objectif}

Le logiciel Bachet a pour but de faciliter la mise en œuvre de la méthode BACHET, en calage comme en simulation. L'objectif visé n'est pas de fournir une boîte noire, difficilement envisageable, mais d'offrir une aide, en automatisant les principales constructions géométriques sur lesquelles repose cette méthode : recherche des caractéristiques de la propagation (atténuation, temps de propagation), tracés d'hydrogrammes atténués ou propagés.

Nous nous sommes limités, pour commencer, au cas le plus simple : il s'agit de prévoir les hydrogrammes de crue à une station donnée, connaissant ces hydrogrammes à une station amont. Le problème des confluents n'est donc pas traité, et les apports intermédiaires diffus sont supposés suffisamment peu importants pour n'avoir pas à être pris en compte explicitement.

\subsection{Méthode choisie}

Sans revenir en détail sur la méthode de Bachet, rappelons qu'elle effectue la prévision d'un hydrogramme aval en appliquant deux transformations successives à l'hydrogramme amont associé : la propagation, l'atténuation. La propagation utilise explicitement une fonction " temps de propagation ", suivant le principe

$Q_{2}\left(t+\Delta t\left(Q_{1}(t)\right)=Q_{1}(t)\right.$.

$Q_{1}$ : hydrogramme amont

$Q_{2}$ : hydrogramme propagé

$Q \rightarrow \Delta t(Q):$ fonction temps de propagation
L'atténuation est obtenue moins automatiquement: elle résulte surtout de l'atténuation "au sommet", calculée au moyen d'une corde d'atténuation fonction du maximum de l'hydrogramme amont (figure 1).

Vis-à-vis de cette méthode dite classique, l'innovation propre à ce logiciel est d'introduire une fonction " atténuation " mise en œuvre de manière tout à fait identique à la fonction "propagation " classique : pour atténuer un hydrogramme, nous utilisons en effet une corde d'atténuation variable, dépendant du débit " en cours " de l'hydrogramme traité, et non plus constante, dépendant du seul débit maximum.

Les justifications sont surtout d'ordre pratique. Pour construire automatiquement un hydrogramme atténué complet (et non plus le seul sommet), il n'est pas évident, et nous ne connaissons pas de justifications théoriques, qu'il soit préférable d'utiliser une corde d'atténuation

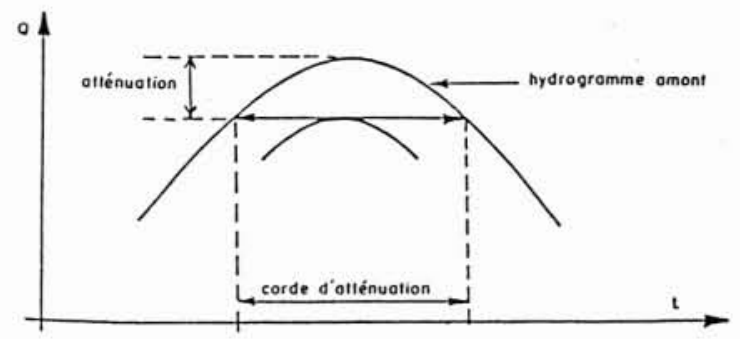

1. Estimation de l'atténuation dans la méthode Bachet classique.

\section{Computerization of the Bachet Method}

With a view to simplifying the use of the Bachet Method, an interactive software has been designed allowing for the adjustment or testing of the method on a series of flood hydrographs. The methodology implemented and computerization of simple geometrical constructions depends on the construction principles of the Bachet rules. The adjustment consists of evaluating two functions known as the "Attenuation Cord " and "Transfer Time", and this can be carried out either on natural hydrographs or converted hydrographs. During the test, the first function converts a upstream hydrograph into an attenuated hydrograph, then the second function converts the latter into a downstream hydrograph. The case concerning these confluents are currently not dealt with. 

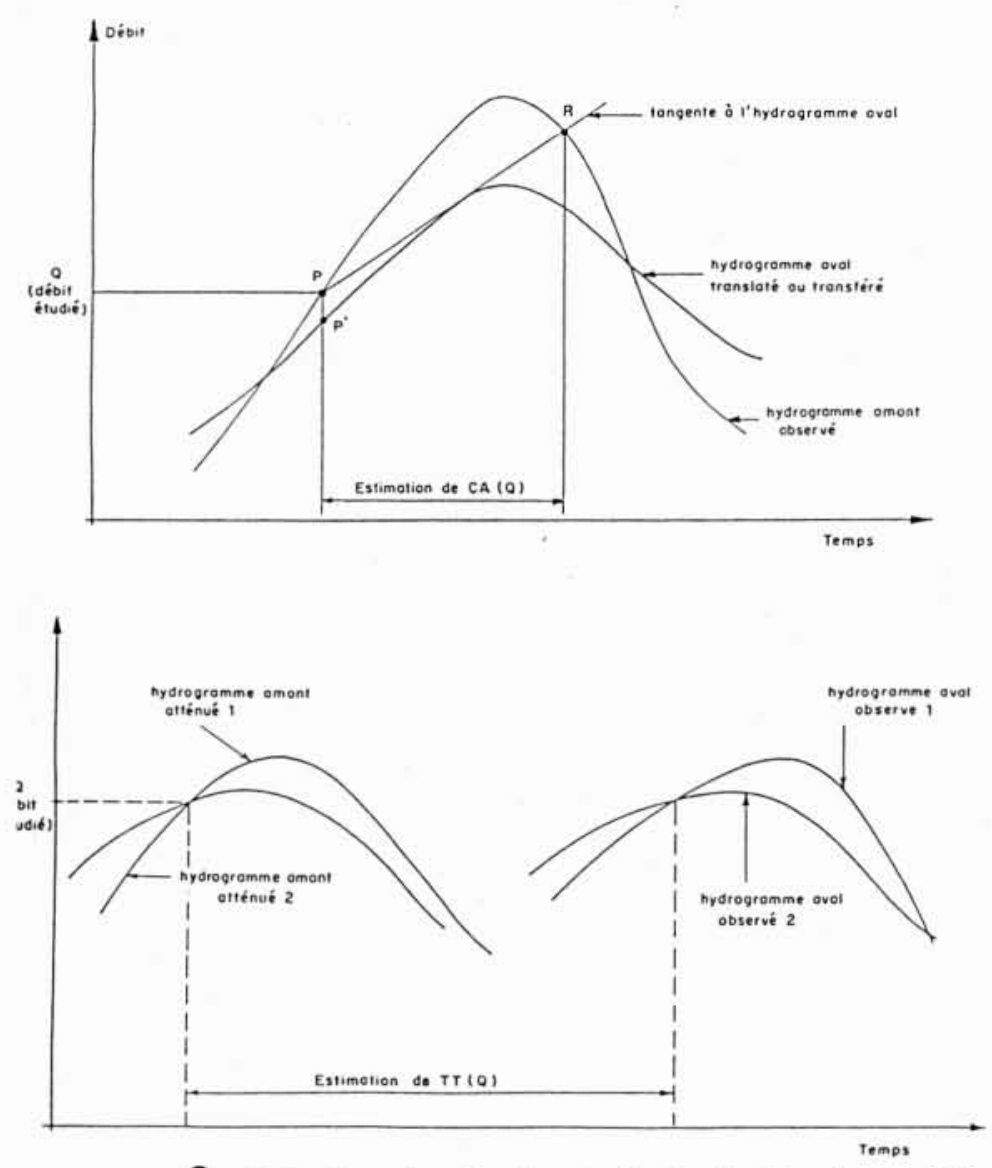

2. Estimation des fonctions "Corde d'atténuation" (CA), "Temps de transfert" (TT).

constante. Or, à partir du moment où elle varie, la manière la plus naturelle est de la faire dépendre du débit à traiter (de façon tout à fait analogue à la fonction propagation), tandis qu'utiliser le seul maximum oblige à introduire alors un type de variation prédéterminé. Ceci reste cependant proposé à titre " expérimental ", et pourra être remis en cause au besoin. Cette procédure est de toute manière peu contraignante :

- en prévision, le programme peut être modifié très aisément pour revenir à la méthode classique;

- en imposant une corde d'atténuation constante au-delà d'un certain seuil (ce qui est souvent le cas dans la méthode classique) les deux méthodes deviennent quasi équivalentes dans le domaine concerné.

Le logiciel traite indépendamment l'atténuation de la propagation, mais une fois donné l'ordre dans lequel ces transformations sont effectuées, il convient de toujours le respecter. Nous avons choisi en premier l'atténuation de manière à estimer la fonction propagation en partant d'hydrogrammes atténuées, situation où la méthode utilisée est la plus valide.

Pour bien marquer ce choix, nous parlerons désormais, pour traiter du phénomène de propagation, de transfert (et non propagation) de l'hydrogramme atténué (le terme propagation étant plus habituel pour parler du " transport" de l'hydrogramme amont naturel).

En résumé, la méthode programmée repose donc sur la donnée de deux fonctions appelées "Corde d'atténuation " (CA), "Temps de transferts " (TT) réalisant les opérations suivantes:

- la fonction CA donne, en partant d'un hydrogramme amont observé un hydrogramme amont atténué; elle traduit le phénomène d'atténuation de la crue, de l'amont vers l'aval;

- la fonction TT donne, à partir d'un hydrogramme amont atténué un hydrogramme aval calculé constituant la prévision de l'hydrogramme aval réel; elle rend compte du temps de propagation de la crue.

Ces deux fonctions sont de la forme $\mathrm{T}=f(\mathrm{Q})$, où $\mathrm{Q}$ est un débit, $\mathrm{T}$ est un temps.

\section{Mise en œuvre informatique}

Le logiciel réalisé n'automatise pas, comme nous l'avons souligné, l'ensemble de la méthode, mais les quatre tâches essentielles : estimation de la fonction CA, estimation de la fonction TT, construction d'un hydrogramme atténué, transfert de cet hydrogramme. Nous présentons maintenant plus en détail chacun de ces travaux.

\subsection{Estimation de la fonction $C A$}

Pour effectuer cette estimation, il faut travailler sur les couples d'hydrogrammes amont observé/amont atténué. Les hydrogrammes amont atténuées étant à priori inconnus, deux cas se présentent :

- si une estimation de la fonction TT est disponible, il suffit de transférer, en sens inverse, les hydrogrammes aval observés;

- si les deux fonctions CA, TT sont inconnues, il faut se contenter d'une simple translation de l'hydrogramme aval, en faisant coïncider, si elles sont cohérentes, les dates des maxima amont/aval.

Ces couples étant ainsi constitués, l'estimation de $C A(Q), Q$ étant donné, est représentée en figure 2, et se résume ainsi :

- recherche du point $P$ associé au débit $Q$ dans l'hydrogramme amont;

- recherche de la tangente à l'hydrogramme aval, issue de $P$, la pente de la tangente est la pente extrémale obtenue en traçant les droites d'intersection successives de l'hydrogramme aval issues de $P$;

- calcul du point $R$, c'est-à-dire de l'intersection tangente/hydrogramme aval;

- $C A(Q)$ est la première coordonnée du vecteur $P R$.

Cette procédure est réalisée pour chaque couple d'hydrogrammes utilisable, et l'estimation retenue est la moyenne des estimations successives obtenues. Enfin la méthode est appliquée pour toute une gamme de débit décrivant, avec un pas de temps suffisamment fin, le domaine de variation étudié. 


\subsection{Estimation de la fonction TT}

Pour effectuer cette estimation, il faut travailler sur les couples d'hydrogrammes amont atténué/aval observé. La difficulté résultant de l'ignorance des hydrogrammes atténués se pose donc de nouveau :

- si une estimation de la fonction $C A$ est disponible, ces hydrogrammes sont calculables;

- sinon, seuls les temps de propagation des maxima des crues sont utilisés.

Si les hydrogrammes atténués sont disponibles, l'estimation de $T T(Q), Q$ étant donné, est représentée en figure 2, et se résume ainsi :

- recherche de tous les hydrogrammes atténués passant par cette valeur;

- pour toute paire d'hydrogrammes ainsi sélectionnés, positionnement relatif des deux courbes de manière à ce que leur intersection se situe en $Q$;

- en effectuant le même positionnement des hydrogrammes aval associés, détermination de l'intersection de ces hydrogrammes aval;

- sous conditions (une seule intersection, même sens de variations des courbes), TT(Q) est alors estimé par le temps séparant les deux intersections.

L'estimation finale de $T T(Q)$ est la moyenne des estimations successives obtenues. Comme précédemment, la méthode est enfin appliquée pour toute une gamme de débits décrivant le domaine étudié.

\subsection{Construction d'un hydrogramme atténué}

Le principe est le suivant :

- à chaque pas de temps, une corde d'atténuation est construite, lorsque cela est possible, sur l'hydrogramme amont; cela suppose, pour une application " temps réel ", une connaissance partielle de cet hydrogramme dans le futur : une corde d'atténuation joint un point $(t, Q(t))$, au point $\left(t^{\prime}=t+C A(Q(t)), Q\left(t^{\prime}\right)\right.$ où $Q$ représente le débit amont; l'hydrogramme est donc supposé connu au point $t+C A(Q(t))$.

- l'hydrogramme atténué est l'enveloppe de toutes les cordes d'atténuation ainsi tracées.

Ce dernier point est le plus délicat à informatiser, compte tenu des cas particuliers toujours possibles.

La procédure retenue, illustrée sur la figure 3 consiste à identifier l'hydrogramme atténué à des segments successifs de corde d'atténuation. En appelant:

$J 1$ le premier l'indice de l'hydrogramme atténué non encore calculé,

ITG l'indice de la dernière corde d'atténuation utilisée,

J2 l'indice de l'extrémité de la corde d'atténuation $I T G$,

la procédure retenue est alors la suivante :

- on recherche la plus proche (en temps) des intersections de la corde ITG, et des cordes tracées de $I T G+1$ à $J 2$;

- soit INTER l'indice obtenu, associé à la corde II;

- de $J 1$ à INTER, l'hydrogramme atténué est identifié

à la corde $I T G$;
- ITG devient $I I, J 1$ devient INTER +1 , et le processus recommence.

Un exemple d'hydrogramme atténué ainsi obtenu est représenté en figure 3 .

\subsection{Transfert d'un hydrogramme atténué}

Cette " opération " est beaucoup plus simple que l'atténuation. Pour calculer la valeur, en un indice $I$, de l'hydrogramme transféré, il suffit de repérer l'indice $I$ de l'hydrogramme initial vérifiant:

$$
I+\operatorname{TRANS}(I) \leqslant J \leqslant(I+1)+\operatorname{TRANS}(I+1)
$$

où TRANS $(I)$ est le temps de transfert associé au débit $Q(I)$. Le débit transféré en $J$ est alors une interpolation des débits $Q(I), Q(I+1)$.

Pour que cette procédure soit toujours cohérente, la recherche de l'indice $I$ doit toujours cohérente, la recherche de l'indice $I$ doit toujours se faire en partant de la valeur obtenue en $J-1$, sans " revenir en arrière ".

\section{Organisation du logiciel}

La conception d'ensemble du logiciel répond à deux soucis :

- simplifier le plus possible la mise en œuvre des méthodes décrites ci-dessus, les intégrer dans un ensemble bien structuré;

- permettre à l'utilisateur de suivre et de contrôler l'ensemble des opérations réalisées.
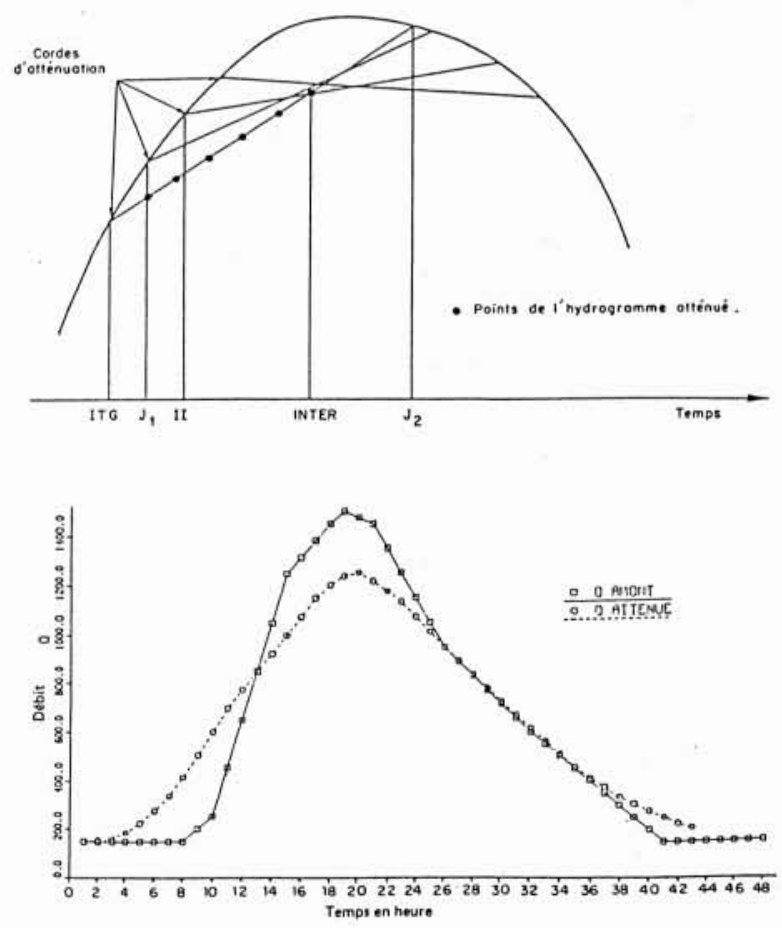

3. Construction d'un hydrogramme atténué. Exemple. 
Le second point justifie le caractère conversationnel, non automatisé, des deux programmes écrits: l'un (BACCAL) effectue l'estimation des fonctions $C A, T T$, l'autre (PREBAC) construit des hydrogrammes atténués ou transférés.

Pour réaliser au mieux cet objectif, l'accent a été mis sur les points suivants :

- standardisation des entrées/sorties, compatibilité totale des deux programmes,

- contrôles a priori des hydrogrammes utilisés,

- contrôles a posteriori des calculs effectués.

Le travail de l'utilisateur, outre son intervention dans ces contrôles, réside dans la manière d'utiliser conjointement les deux programmes, et dans le travail d'analyse des résultats. Cela résulte essentiellement de l'« l'imbrication " des deux fonctions $C A, T T$ : une estimation correcte de $T T$ nécessite la construction des hydrogrammes atténués, et se trouve donc liée à l'estimation de $C A$, et inversement, l'estimation correcte de $C A$ est liée à celle de $T T$.

La démarche souhaitable nous semble la suivante:

- d'abord, estimer chacune des deux fonctions $C A, T T$ à partir des hydrogrammes amont/aval observés;

- à partir de ces deux fonctions, en utilisant le programme PREBAC, construire les hydrogrammes amont atténués et aval transférés;

- refaire une nouvelle estimation des fonctions $C A, T T$ en utilisant cette fois ces hydrogrammes construits;

- comparer à la main les deux estimations, voir quels phénomènes expliquent les différences; en fonction de cette analyse éliminer éventuellement un certain nombre de crues ou d'estimations, et aboutir ainsi à des estimations vraisemblables de ces fonctions;

- reprendre les itérations (estimations $\rightarrow$ construction d'hydrogrammes $\rightarrow$ estimations) pour regarder s'il y a ou non " convergence"; si oui, les performances de la méthode en prévision (programme PREBAC) doivent alors être étudiées pour valider définitivement ce calage; si non, un travail plus spécifique sera nécessaire, notamment pour déterminer si la méthode Bachet en elle-même n'est pas applicable, ou bien si la mise en œuvre « technique " de cette méthode est seule en cause.

\section{Conclusion}

Le logiciel Bachet est un premier essai d'informatisation de la méthode Bachet. Compte tenu du caractère largement empirique de cette méthode, les programmes écrits ne sauraient être figés : ils devront être testés sur des cas d'application aussi variés que possible, et en fonction des résultats de ces expérimentations, certains choix de méthodes pourront être revus, ou certains développements ajoutés. Ce logiciel s'intègre d'autre part dans la bibliothèque informatique "Aide à la prévision de crues ", mise en place progressivement sous l'impulsion du ministère de l'Environnement, et son utilisation s'en trouve donc plus aisée. Cela devrait permettre de comparer plus souvent les résultats, souvent intéressants, à ceux de méthodes plus largement utilisées, comme les modèles linéaires.

Adresse des auteurs

Messieurs J. Miquel et A. Lebossé

EDF. Laboratoire national d'hydraulique

6, quai Watier

78400 Chatou

Tél. : 30.71.78.21 
M. MOREL-SEYTOUT: Je ne connais pas la méthode Bachet, mais mon impression est que cette méthode graphique est une fausse méthode des caractéristiques. Je présume que $\mathrm{M}$. Bachet développerait à notre époque une méthode beaucoup plus analytique qui serait basée sur le traitement numérique d'une équation de convection-diffusion; sa méthode serait alors probablement une méthode basée sur l'éclatement d'opérateurs avec une étape de convection linéaire par caractéristique et une étape de diffusion, l'ensemble étant éventuellement inscrit dans un processus itératif pour traiter les non linéarités. Cette approche de fausses caractéristiques, si elle était programmée, insufflerait une nouvelle jeunesse à la méthode Bachet ce qui ne me paraît pas le cas avec la programmation de l'approche initiale purement graphique qui a été présentée ici. (Texte reconstitué d'après des notes prises en séance).

M. LEBOSSE: L'un n'empêche pas l'autre et le Laboratoire National d'Hydraulique a d'ailleurs une longue expérience dans les méthodes numériques utilisant la théorie des caractéristiques, il a par exemple développé une méthode originale comme la convection faible à base de caractéristiques. Mais il faut replacer cette étude dans son cadre : il s'agit ici d'offrir aux utilisateurs de la méthode Bachet, qui sont nombreux, un outil graphique informatisé qui les décharge des tâches manuelles fastidieuses et leur permette de maintenir les réglettes Bachet; en effet dans de nombreux cas les réglettes sont anciennes, les lits des cours d'eau ont évolué et l'outil présenté ici doit justement faciliter la remise à jour des réglettes.

M. MOREL-SEYTOUT: Ma réflexion portait aussi, en tant qu'enseignant, sur l'aspect plus formateur qu'aurait une approche analytique par rapport à une approche graphique dans la promotion de cette méthode (notes prises en séance).

M. le Président: Deux points me paraissent importants à souligner dans la méthode Bachet car ils lui apportent toute sa spécificité. C'est, d'une part, le remplacement d'une dérivée seconde en espace par une dérivée seconde en temps (au lieu d'avoir besoin de trois points en espace, on utilisera trois points en temps); c'est, d'autre part, l'estimation d'une dérivée seconde en espace par une corde, c'est-à-dire par une construction géométrique. C'est donc une méthode très originale dans son traite- ment du terme diffusif; elle offre en outre l'avantage pour le prévisionniste d'être l'une des rares méthodes qui travaillent en hauteur; c'est donc une méthode à ne pas négliger d'un point de vue pratique.

M. THIRRIOT: Je voudrais souligner l'extrême vitalité de la méthode Bachet dégagée par les remarques des professeurs Morel-Seytout et Bocquillon. Cette méthode, loin d'être une recette de cuisine, apparaît comme une application précoce des méthodes asymptotiques: le terme de diffusion joue ici le rôle d'une perturbation singulière sur la solution de l'équation hyperbolique convective. Il faut aussi souligner que cette méthode prend en compte les non linéarités, c'est là quelque chose de très important; d'autre part l'emploi de la technique de la corde pour approximer les dérivées secondes a un effet stabilisant lorsqu'on traite une crue réelle, alors que les bruits effaceraient complètement le signal structuré de la crue si l'on calculait directement une dérivée seconde de manière discrète. Bachet est enterré mais il n'est pas mort !

M. P.A. ROCHE : La discussion qui vient d'avoir lieu confirme l'intérêt métholodogique de la méthode Bachet (notamment l'effet de lissage obtenu grâce à l'utilisation des cordes pour estimer des dérivées secondes). Ce débat avait déjà eu lieu lorsqu'il s'est agi de décider de programmer la méthode, et J. Miquel avait à l'époque pris en compte ces différents éléments.

M. BANAL: Le fait que la méthode Bachet ait été utilisée pour toute une série de bassins donc tarée par l'expérience confère-t-il à sa transposition informatique un intérêt particulier ?

M. P.A. ROCHE: Le principal acquis réside dans l'expérience accumulée sur la dispersion que l'on peut accepter sur les nuages de points utilisés pour les calages. On sait de ce fait assez bien détecter les anomalies et les corriger; cela représente effectivement un plus pour cette méthode par rapport à d'autres.

M. MOREL-SEYTOUT: Je suis d'accord avec M. Thirriot qu'il faut tirer son chapeau à $M$. Bachet; c'était un précurseur qui a utilisé les moyens de son temps. De même en programmation dynamique, M. Masse fut un précurseur mais c'est à Bellman qu'on attribue aujourd'hui la programmation dynamique, car cette méthode a été popularisée par cet auteur qui disposait de l'ordinateur. (notes prises en séance). 


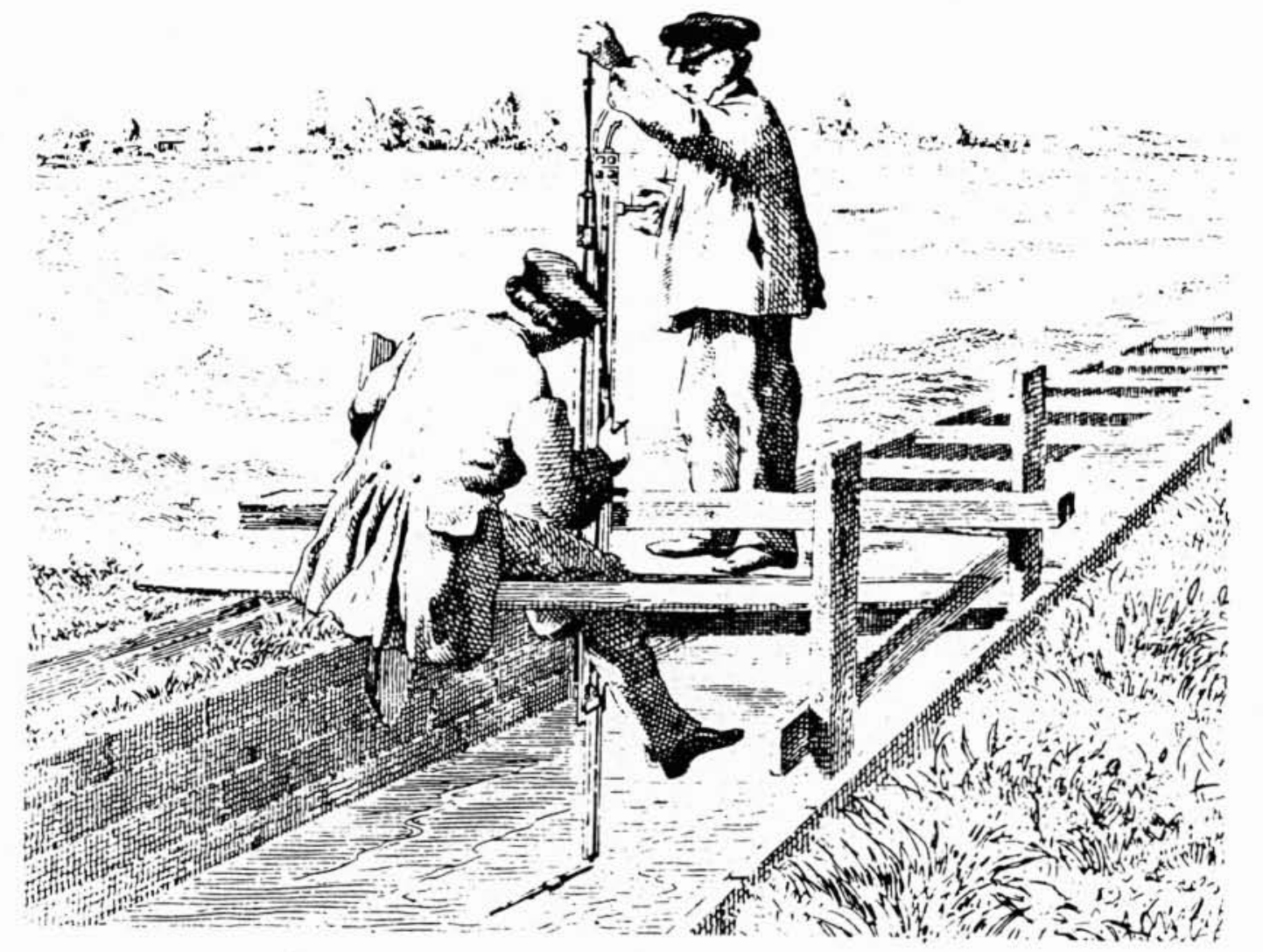

